\title{
Synergistic antitumor activity of aspirin and erlotinib: Inhibition of p38 enhanced aspirin plus erlotinib-induced suppression of metastasis and promoted cancer cell apoptosis
}

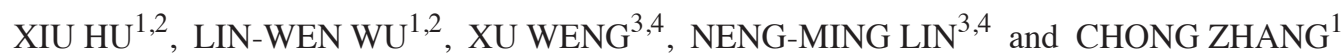 \\ ${ }^{1}$ School of Medicine, Zhejiang University City College, Hangzhou, Zhejiang 310015; \\ ${ }^{2}$ College of Pharmaceutical Sciences, Zhejiang University, Hangzhou, Zhejiang 310058; \\ ${ }^{3}$ Department of Clinical Pharmacology; ${ }^{4}$ Hangzhou Translational Medicine Research Center, \\ Hangzhou First People's Hospital, Nanjing Medical University, Hangzhou, Zhejiang 310006, P.R. China
}

Received November 21, 2017; Accepted May 31, 2018

DOI: $10.3892 / \mathrm{ol} .2018 .8956$

\begin{abstract}
High-dose erlotinib is effective for non-small cell lung cancer patients with brain metastases. The aim of the present study was to investigate whether aspirin could increase the anti-proliferative and anti-metastatic effects of regular erlotinib treatment. The data demonstrated that combining aspirin with erlotinib significantly induced apoptosis and inhibited tumor cell proliferation in several human cancer types. Furthermore, aspirin plus erlotinib significantly induced the activation of E-cadherin and suppression of $\mathrm{p} 38$. The data also indicated that the p38/E-cadherin pathway may be involved in the apoptosis caused by the combination of aspirin and erlotinib. As p38 and E-cadherin also serve a key role in epithelial-to-mesenchymal transition (EMT) and cancer metastasis, we hypothesized that the combination of aspirin and erlotinib may significantly inhibit tumor metastasis. First, aspirin plus erlotinib achieved
\end{abstract}

Correspondence to: Dr Chong Zhang, School of Medicine, Zhejiang University City College, 51 Huzhou Street, Hangzhou, Zhejiang 310015, P.R. China

E-mail: zhangchong@zucc.edu.cn

Professor Neng-Ming Lin, Department of Clinical Pharmacology, Hangzhou Translational Medicine Research Center, Hangzhou First People's Hospital, Nanjing Medical University, 261 Huansha Road, Hangzhou, Zhejiang 310006, P.R. China

E-mail:1nm1013@163.com

Abbreviations: CI, combination index; PI, propidium iodide; Ab, antibody; EMT, epithelial to mesenchymal transition; MAPKs, mitogen-activated protein kinases; PI3K, phosphatidylinositol 3-kinase; EGFR-TKI, epidermal growth factor receptor tyrosine kinase inhibitor; V, tumor volume; RTV, relative tumor volume; HUVEC, human umbilical vein endothelial cells; CAM, chick embryo chorioallantoic membrane; GSK-3 $\beta$, glycogen synthase kinase-3 $\beta$; NF- $\kappa \mathrm{B}$, nuclear factor- $\kappa \mathrm{B}$.

Key words: aspirin, erlotinib, combination, E-cadherin, p38 potent inhibition of cancer cell migration and invasion, which are crucial for cancer metastasis. Next, the results demonstrated that aspirin plus erlotinib inhibited angiogenesis by suppressing endothelial cell migration and invasion. Moreover, it was confirmed that aspirin plus erlotinib exerted synergistic anti-angiogenic effects. Finally, the synergistic anti-proliferative and anti-metastatic effects of the combination of aspirin with erlotinib were further validated in an A549 xenograft model in vivo. In conclusion, aspirin plus erlotinib may be an effective combination regimen for patients with metastatic cancer.

\section{Introduction}

The severity and incurability of cancer are attributed to cancer metastasis, the initiation of which requires enhanced migratory and invasive capabilities (1). Tumor cells undergo epithelial-to-mesenchymal transition (EMT) by inducing the expression of mesenchymal markers and inhibiting the expression of epithelial markers during tumor progression, and EMT enables cancer cells to invade and metastasize (2). EMT is a process characterized by the decrease of E-cadherin expression and the increase of the expression of mesenchymal markers, such as vimentin, $\beta$-catenin and N-cadherin (3). Thus, E-cadherin suppresses metastasis and its inhibition promotes the development of malignant epithelial cancers (4). In addition, EMT may render cancer cells resistant to various chemotherapeutic/targeted agents via reducing apoptotic sensitivity (5). EMT attenuates the activation of caspase- 8 via DR4/DR5 accompanied by E-cadherin inhibition. E-cadherin interacts with DR4/DR5, thereby promoting caspase-8 activation and apoptosis (6). Thus, E-cadherin has potentially significant biological implications in the cross-regulation between EMT and apoptosis (7).

Several transcription factors, such as ZEB and Slug, can repress E-cadherin $(8,9)$. In addition, the expression of these transcription factors is controlled by a complex network of signaling molecules, including mitogen-activated protein kinases (MAPKs), glycogen synthase kinase-3 $\beta$ (GSK-3 $\beta$ ), phosphatidylinositol 3-kinase (PI3K) and nuclear factor $\kappa \mathrm{B}(\mathrm{NF}-\kappa \mathrm{B})(10)$. MAPKs (ERK, JNK and p38) can 
promote EMT and tumor cell metastasis $(11,12)$. p38 activation is required for EMT, accompanied by E-cadherin downregulation (13). Furthermore, p38 plays a dual role in chemotherapeutic agent-induced apoptosis (14). Several chemotherapeutic agents require p38 to induce apoptosis (15). However, p38 can also mediate resistance to apoptosis, as p38 activation results in induction of cyclooxygenase-2 (COX-2) overexpression, which triggers resistance to apoptosis in cancer cells $(16,17)$.

Aspirin is a non-selective COX inhibitor and has been used as an anti-inflammatory drug for $>100$ years (18). Regular use of aspirin is effective in preventing several common cancers, including colon, breast, liver and lung cancer (19-22). Aspirin acts by targeting several tumor cell functions, including migration, and has been reported to reduce the risk of cancer initiation and progression $(1,23)$. Aspirin modulates matrix metalloproteinase-2 (MMP-2) and E-cadherin production and, therefore, possesses anti-metastatic properties (24). Erlotinib, an epidermal growth factor receptor tyrosine kinase inhibitor (EGFR-TKI), blocks the autophosphorylation of EGFR and suppresses cell proliferation, along with induction of apoptosis and anti-angiogenic effects, inhibiting invasion and metastasis $(25,26)$. In the present study, we hypothesized that the combination of aspirin with erlotinib may exert synergistic antitumor effects by inhibiting cancer cell proliferation and metastasis. First, our data demonstrated that aspirin combined with erlotinib exerted a synergistic anti-proliferative effect and promoted apoptosis in multiple human cancer cells. Furthermore, we also found that the combination of aspirin and erlotinib was significantly more effective in inhibiting cancer cell migration and invasion, which are crucial for cancer metastasis. In addition, the synergistic anti-angiogenic effects of aspirin plus erlotinib were confirmed in vitro and in vivo. Finally, the synergistic anti-proliferative and anti-metastatic effects of the combination of aspirin with erlotinib were further validated in an A549 xenograft model in vivo. These findings suggested that aspirin plus erlotinib may be an efficient combination regimen for patients with metastatic cancer.

\section{Materials and methods}

Materials. Aspirin was purchased from Sigma-Aldrich (St. Louis, MO, USA) and erlotinib was obtained from LC Laboratories (Woburn, MA, USA). SB-203580, a p38 inhibitor, was obtained from Selleck Chemicals (Houston, TX, USA).

Cell culture. Human lung carcinoma cell lines (NCI-H1299 and A549), ovarian carcinoma cell line (HO-8910), colon carcinoma cell line (HCT-116) and gastric carcinoma cell line (SGC-7901) were obtained from Shanghai institute of biochemistry and cell biology. A549 was maintained in Ham's F12 medium $+10 \%$ fetal bovine serum (FBS). SGC-7901 was maintained in RPMI-1640 medium $+10 \%$ FBS. HO-8910, NCI-H1299 and HCT-116 were maintained in $\mathrm{DMEM}+10 \% \mathrm{FBS}$.

Sulphorhodamine (SRB) cytotoxicity assay. The cytotoxic activity was measured by the SRB method, as previously described (27).
Colony-forming assay. Cancer cells (500-1,000 cells/dish) were plated into six-well plates, treated with drugs every 3-4 days for 2 weeks, and then stained by crystal violet (28).

Analysis of apoptosis and determination of mitochondrial membrane depolarization. Cancer cells (3x105/well) were exposed to the drugs, harvested and washed with PBS. Then, propidium iodide (PI) staining was used to detect apoptosis, and the mitochondrial membrane depolarization was determined by 5,5',6,6'tetrachloro-1,1',3,3'-tetraethylbenzimidazol-carbocyanine iodide (JC-1) staining as described previously (27).

Protein preparation from tissue and cell samples and western blot analysis. The western blotting was performed as described previously (29). The mouse antibodies used for western blotting were obtained from different resources: Anti- $\beta$-actin monoclonal antibody $(\mathrm{Ab})$ (from BD Biosciences, Franklin Lakes, NJ, USA) and anti-XIAP monoclonal Ab (Santa Cruz Biotechnology, Dallas, TX, USA). The rabbit antibodies used for western blotting were purchased from different resources: anti-PARP polyclonal $\mathrm{Ab}$, anti-procaspase-3 polyclonal $\mathrm{Ab}$, anti-Mcl-1 polyclonal $\mathrm{Ab}$ and anti-p38 polyclonal Ab (Santa Cruz Biotechnology), anti-phospho-p38 (Thr-180/Tyr-182) polyclonal $\mathrm{Ab}$ and anti-E-cadherin polyclonal $\mathrm{Ab}$ (Cell Signaling Technology, Danvers, MA, USA).

Wound-healing assay. Cells were seeded in 24-well plates and cultured until they reached confluence. Confluent monolayer cells were gently scratched with a sterile pipette tip and then washed three times with PBS to clear cell debris and suspended cells. Fresh serum-free medium was added, and the cells were allowed to close the wound for $48 \mathrm{~h}$ under normal conditions. Images of the wound in the same relative position were captured with a computer-assisted microscope (Olympus, Tokyo, Japan).

Cell invasion assay. Cell invasion experiments were performed using 24-well modified Boyden chambers (Costar, NY, USA) containing a polycarbonate membrane with $8.0-\mu \mathrm{m}$ pores according to the manufacturer's instructions. First, the membranes were coated with $25 \mu \mathrm{g}$ Matrigel (BD Biosciences). Cells were seeded at a density of $1 \times 10^{6}$ cells/well in the upper chamber with culture medium $(200 \mu \mathrm{l})$ alone, while the bottom of the plate was filled with culture medium (500 $\mu \mathrm{l})$ supplemented with $20 \%$ FBS. Cells that invaded the underside of the membrane were fixed in $1 \%$ methanol and stained by crystal violet.

Chick embryo chorioallantoic membrane (CAM) assay. Inhibition of angiogenesis was determined using the CAM assay. Fertilized chicken eggs were incubated at $37^{\circ} \mathrm{C}$ in a $50 \%$ humidified atmosphere. On day 7 , the eggshell was cracked and gently opened into the plate to avoid any unnecessary physical stress. It was made sure that the yolk sac membrane remained intact and that the embryo was viable. Then, a sterile filter paper square saturated with aspirin, erlotinib or their combination was placed in areas between vessels, but not onto any large vessels. After a 48-h incubation, the membranes were examined by microscopy and photographic documentation. Angiogenesis was quantified by 

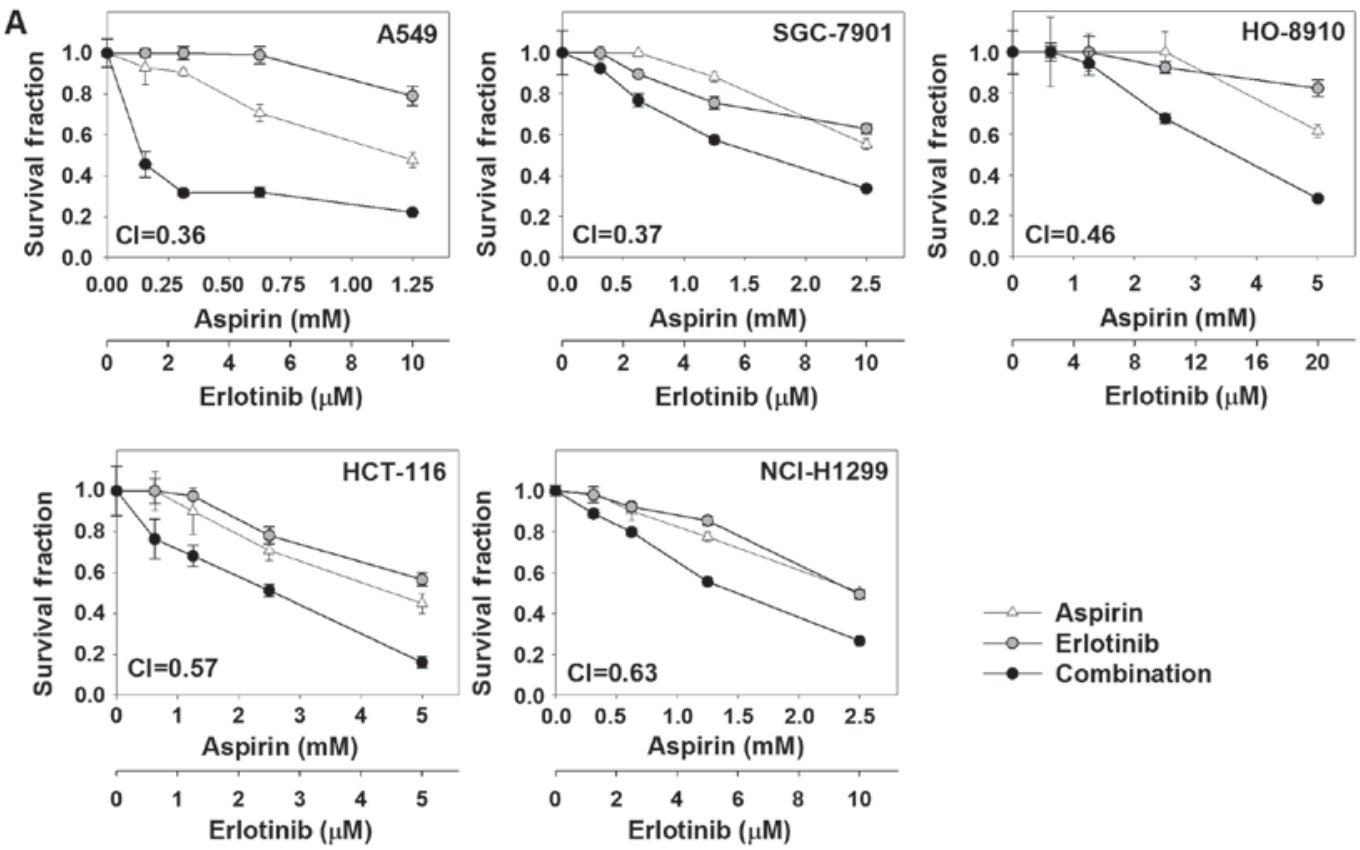

B
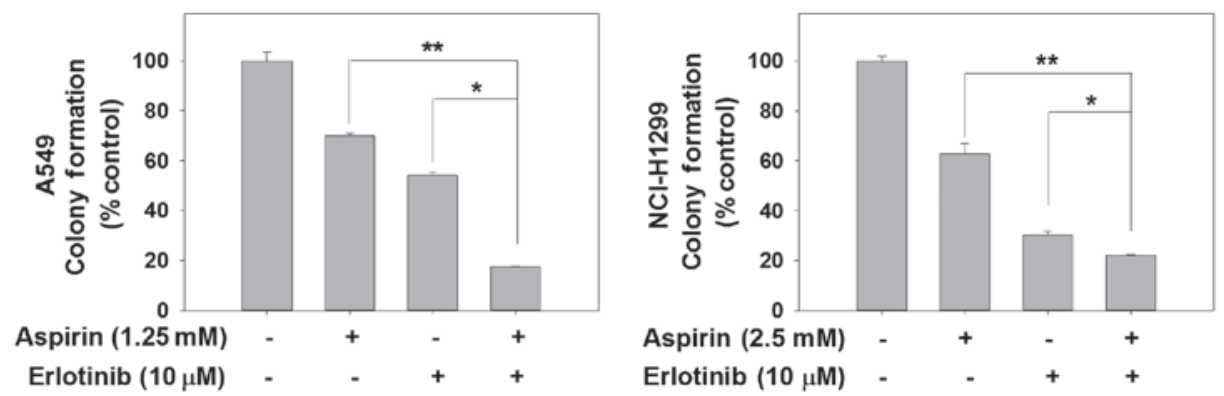

Figure 1. The cytotoxicity of aspirin plus erlotinib in carcinoma cells. (A) The carcinoma cells were treated with indicated drugs for $72 \mathrm{~h}$. The cytotoxicity of aspirin, erlotinib or the combination were showed. (B) A549/NCI-H1299 cells were incubated with drugs for 14 days, and then stained with crystal violet. " $\mathrm{P}<0.05$ and ${ }^{* * *} \mathrm{P}<0.01$.

counting blood vessel branches; at least 10 viable embryos were tested for each treatment.

Plasmids transfection. Cells $\left(3 \times 10^{4}\right)$ were seeded in 6-well plates. E-cadherin (RG220731; Origene Technologies, Rockville, MD, USA) and empty vector plasmid were transfected into cells using Lipofectamine 3000 (Invitrogen; Thermo Fisher Scientific, Inc., Waltham, MA, USA) according to the manufacturer's instructions.

siRNA transfection. Cells $\left(5 \times 10^{4}\right)$ were seeded in 6-well plates. P38 siRNA and control siRNA (Genepharma, Shanghai, China) were transfected into cells using Oligofectamine reagent (Invitrogen; Thermo Fisher Scientific, Inc.) according to the manufacturer's instructions. Sense p38 siRNA sequence was 5'-GCAUAAUGGCCGAGCUGUUTT-3'.

Antitumor activity in vivo and histopathological evaluation of tumor metastasis. A549 xenografts were performed as previously described (29). BALB/c (nu/nu) mice were maintained under sterile conditions using an individually ventilated cage system, randomized to 4 groups and then treated with vehicle, aspirin (100 mg/kg, i.g. administration) daily and/or erlotinib $(20 \mathrm{mg} / \mathrm{kg}$, i.p. administration) twice per week for 29 days $(n=6)$. Finally, the mice were sacrificed at the end of the treatment. The liver were fixed in $10 \%$ buffered formalin and embedded in paraffin, $5-\mu \mathrm{m}$ tissue sections were stained with hematoxylin and eosin (H\&E). All animal handling was performed in accordance with the National Institutes of Health Guide for the Care and Use of Laboratory Animals, and approved by the Zhejiang University City College Animal Care and Use Committee (Hangzhou, Zhejiang, China).

TUNEL staining. TUNEL assay was done using TUNEL apoptosis assay kits (Beyotime Institute of Biotechnology, Shanghai, China) as recommended by the manufacturer.

Statistical analyses. One-way ANOVA followed by Tukey's post hoc test and Two-tailed student's t-tests were used to examine the significance of differences among groups. Data points in graphs represent the mean $\pm \mathrm{SD}\left({ }^{*} \mathrm{P}<0.05 ;{ }^{* *} \mathrm{P}<0.01\right)$. For $\mathrm{SRB}$ assay, combination index $(\mathrm{CI})$ values were calculated using Calcusyn (Biosoft, Great Shelford, Cambridge, UK) and the mean CI values were chosen for presentation. A CI value $<0.9$ indicated synergism; 0.9 to 1.10 , additive; and $>1.10$, antagonism.

\section{Results}

Cytotoxicity of aspirin plus erlotinib in human carcinoma cell lines. First, we assessed the anticancer activity of the 

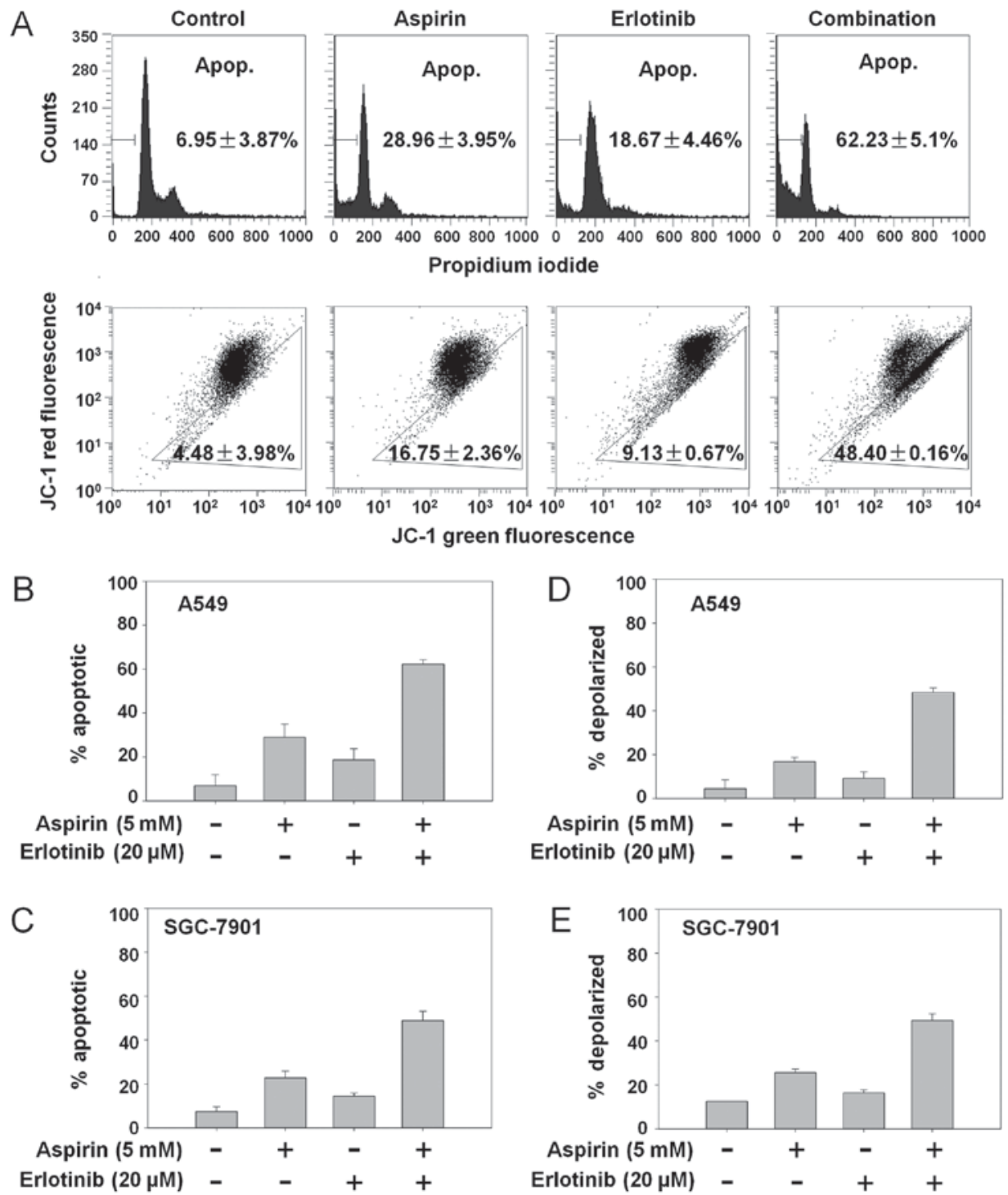

Figure 2. Aspirin plus erlotinib induced apoptosis via mitochondrial pathway. (A) A549 cells were incubated with aspirin $(5 \mathrm{mM})$, erlotinib (20 $\mu \mathrm{M})$ or the combination for $48 \mathrm{~h}$, and then cells were stained with PI (top panel)/JC-1 (bottom panel) followed by flow cytometry detection. (B and C) Cancer cells in 6-well plates were treated with drugs for $48 \mathrm{~h}$ and detected by flow cytometry after PI staining. (D and E) Cancer cells were treated with drugs for $48 \mathrm{~h}$ and detected by flow cytometry after JC-1 staining. PI, propidium iodide; JC-1, 5,5',6,6'tetrachloro-1,1',3,3'-tetraethylbenzimidazol-carbocyanine iodide.

combination of aspirin and erlotinib by SRB assay in 5 human carcinoma cell lines. The survival curves for aspirin and/or erlotinib are shown in Fig. 1A. We found that aspirin plus erlotinib significantly reduced the survival fraction in human cancer cells compared with each agent alone. To verify the synergistic anticancer effect of aspirin and erlotinib, CI values were calculated. Aspirin plus erlotinib exerted synergistic cytotoxic effects on 5 human carcinoma cell lines (CI values $<0.7)$. In a long-term colony-forming assay, the combination of aspirin and erlotinib resulted in significant inhibition on the proliferation of A549 and NCI-H1299 cells, while monotherapy induced a moderate inhibition $(\mathrm{P}<0.01$, ANOVA) (Fig. 1B). Taken together, these finding indicate that the combination of aspirin and erlotinib was more effective in limiting colony formation and cell growth of human cancer cells in vitro compared with either agent alone.

Aspirin plus erlotinb induces mitochondrial mediated apoptosis via p38/ E-cadherin pathway. We first investigated whether the synergistic anticancer effects of aspirin plus erlotinib were related to the induction of apoptosis. The percentage of apoptotic A549 cells was $6.95 \%$ in the control group, $28.96 \%$ with aspirin treatment, $18.67 \%$ with erlotinib treatment, and $62.23 \%$ in the aspirin plus erlotinib group (Fig. 2A, top panel). Aspirin plus erlotinib significantly enhanced apoptosis in both A549 and SGC-7901 cells compared with either drug alone ( $\mathrm{P}<0.01$, ANOVA; Fig. 2B and $\mathrm{C})$. Next, we investigated whether aspirin plus erlotinib affected the mitochondrial membrane potential. As shown in Fig. 2A (bottom panel), $\mathrm{D}$ and $\mathrm{E}$, aspirin plus erlotinib increased the percentage of mitochondrial membrane depolarized carcinoma cells compared with either drug alone ( $\mathrm{P}<0.01$, ANOVA). Thus, our data suggested that the synergistic effects of aspirin plus erlotinib are mediated via the mitochondrial apoptotic pathway. In addition, aspirin plus erlotinib markedly induced PARP cleavage and XIAP suppression in two of the cancer cell lines (Fig. 3A). The induction of EMT and activation of $\mathrm{p} 38$ are associated with resistance to erlotinib, and blockade of $\mathrm{p} 38$ 
A

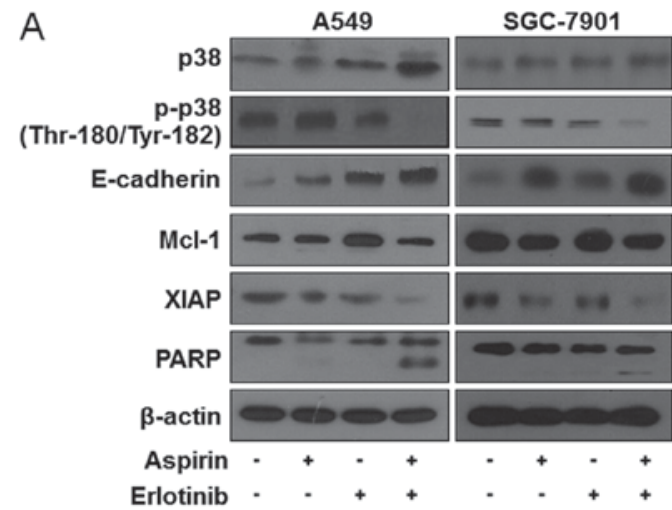

B

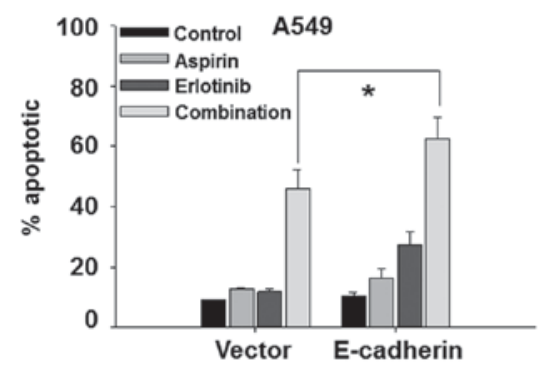

C

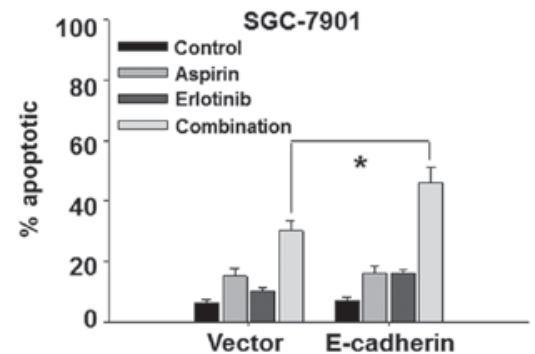

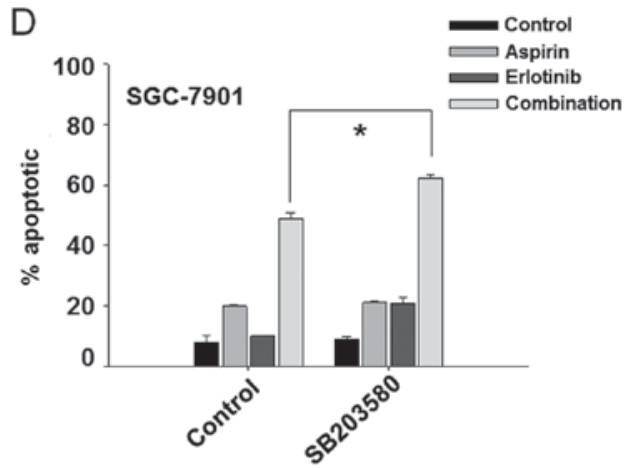

$\mathrm{E}$
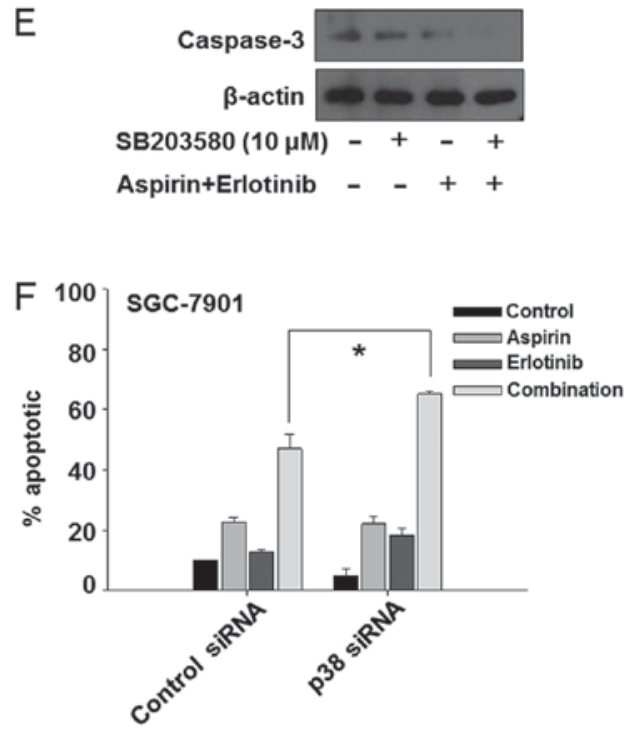

Figure 3. The effect of aspirin plus erlotinib on p38/E-cadherin pathway. (A) A549/SGC-7901 cells were incubated with aspirin (5 mM), erlotinib (20 $\mu \mathrm{M}$ ) or the combination for $24 \mathrm{~h}$, and then the protein expressions were detected by western blot assay. A549 (B) and SGC-7901 (C) cells were transfected with E-cadherin plasmid, and then treated with aspirin $(5 \mathrm{mM})$, erlotinib $(20 \mu \mathrm{M})$ or the combination for $48 \mathrm{~h}$. (D) SGC-7901 cells were pre-incubated with $10 \mu \mathrm{M}$ SB-203580 for $1 \mathrm{~h}$ and then incubated with $5 \mathrm{mM}$ aspirin and/or $20 \mu \mathrm{M}$ erlotinib for $48 \mathrm{~h}$. PI staining was used to detect apoptosis. (E) SGC-7901 cells were pre-incubated with $10 \mu \mathrm{M} \mathrm{SB}-203580$ for $1 \mathrm{~h}$ and then incubated with $5 \mathrm{mM}$ aspirin plus $20 \mu \mathrm{M}$ erlotinib for $48 \mathrm{~h}$. The expression of caspase- 3 was examined. (F) SGC-7901 cells were transfected with p38 siRNA and control siRNA, and then treated with aspirin $(5 \mathrm{mM})$, erlotinib (20 $\mu \mathrm{M})$ or the combination for $48 \mathrm{~h}$. PI staining was used to detect apoptosis. "P<0.05. PI, propidium iodide.

was found to be able to suppress EMT in erlotinib-resistant cancer cells (30). Furthermore, the upregulation of E-cadherin increased the sensitivity of several TKIs, such as gefitinib and erlotinib $(31,32)$. Thus, we were interested in examining the involvement of p38 and E-cadherin in the aspirin and erlotinib combination treatment. Interestingly, as shown in Fig. 3A, the enhanced apoptosis induced by aspirin plus erlotinib was accompanied by $\mathrm{p} 38$ inhibition and overexpression of E-cadherin in A549 and SGC-7901 cells, indicating that aspirin may reverse erlotinib resistance via the p38/E-cadherin pathway. To further investigate the involvement of the p38/E-cadherin pathway in the synergistic effects of aspirin and erlotinib, we first performed E-cadherin overexpression experiments by transfecting cancer cells with an E-cadherin plasmid. As shown in Fig. 3B and C, overexpression of E-cadherin increased the apoptosis induced by aspirin plus erlotinib in A549 and SGC-7901 cells. Next, a p38 inhibitor (SB-203580) was found to increase the apoptosis induced by aspirin plus erlotinib in SGC-7901 cells (Fig. 3D and E). Moreover, p38 depletion by siRNA also enhanced aspirin plus erlotinib-induced apoptosis (Fig. 3F). Therefore, these data indicated that the $\mathrm{p} 38 / \mathrm{E}$-cadherin pathway may be involved in the enhanced apoptosis induced by aspirin plus erlotinib treatment.

Aspirin plus erlotinib inhibits invasion and migration of human carcinoma cells. E-cadherin inhibition is an important step in EMT and a hallmark of metastatic cells (33). Our data already demonstrated that aspirin plus erlotinib could increase the expression of E-cadherin; thus, we hypothesized that aspirin plus erlotinib may inhibit EMT and cancer metastasis. EMT enables cancer cell migration and invasion, and a scratch assay was conducted on A549 and SGC-7901 cells to assess cell migration, which is a defining feature of the mesenchymal phenotype. As shown in Fig. 4A (top panel), untreated A549 cells migrated within $24 \mathrm{~h}$ after wounding, whereas there was moderate inhibition of cell migration when the A549 cells were treated with erlotinib at $2.5 \mu \mathrm{M}$. However, the suppressive effect on migration was most prominent in A549 cells treated with aspirin plus erlotinib. Similar results were 

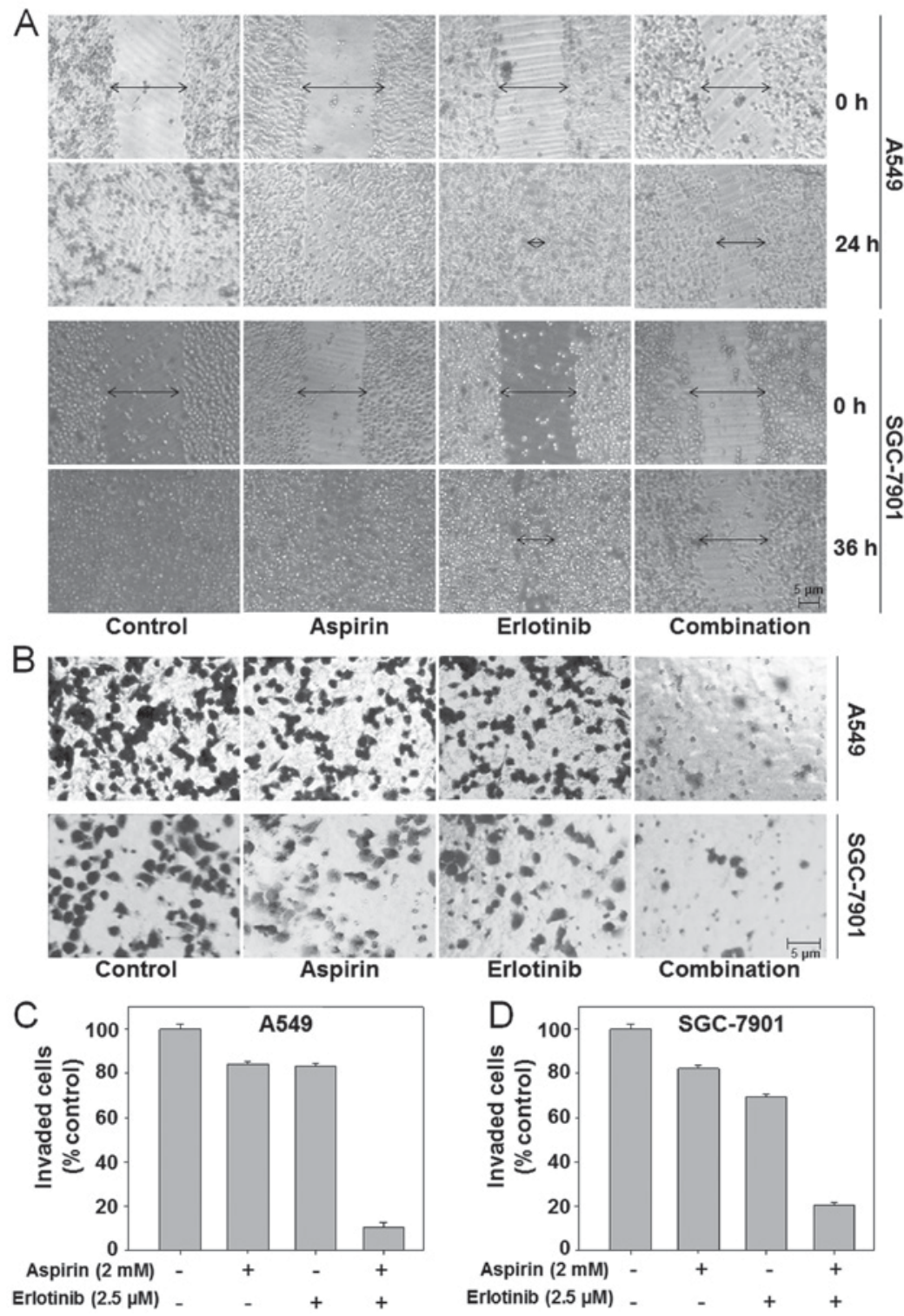

Figure 4. Aspirin plus erlotinib inhibited invasion and migration of cancer cells. (A) Cancer cells were gently scratched with a sterile pipette tip and then washed with PBS three times. Next, cancer cells were incubated with $2 \mathrm{mM}$ aspirin, $2.5 \mu \mathrm{M}$ erlotinib or the combination for indicated times. Photographs were taken by microscope. (B) Cancer cells were seeded in the upper chamber and then cells were incubated with $2 \mathrm{mM}$ aspirin, $2.5 \mu \mathrm{M}$ erlotinib or the combination for $24 \mathrm{~h}$. Cancer cells invaded the underside of the membrane were fixed in $1 \%$ methanol and stained by crystal violet. Quantification of invaded A549 (C) and SGC-7901 (D) cells were counted in Matrigel Transwell invasion assays.

observed in SGC-7901 cells (Fig. 4A, bottom panel). In addition, the effects of aspirin plus erlotinib on cancer cell invasion were evaluated by Matrigel Transwell invasion assays. Our data demonstrated that aspirin or erlotinib achieved moderate inhibition of cell invasion in the A549 and SGC-7901 cell lines. However, aspirin plus erlotinib led to a significant reduction in the number of invading cancer cells compared with the effects of either aspirin or erlotinib alone $(\mathrm{P}<0.01$, ANOVA) (Fig. 4B-D). Our results indicated that the combination of aspirin and erlotinib enhanced the inhibition of cancer cell migration and invasion compared with either drug alone.

Synergistic inhibition of angiogenesis by aspirin plus erlotinib. Endothelial cells can invade the surrounding basement membrane, and then migrate into the stroma during tumor angiogenesis. Finally, endothelial cells organize to form new capillaries, which are crucial for tumor metastasis (34).
To evaluate the anti-angiogenic function of the aspirin plus erlotinib combination, we first detected its effects on migration and invasion of human umbilical vein endothelial cells (HUVECs). There was no inhibition of cell migration when HUVECs were treated with aspirin or erlotinib alone. However, when the two drugs were combined, the inhibitory effect on HUVEC migration was enhanced in the scratch assay (Fig. 5A). Similarly, aspirin plus erlotinib treatment induced a substantial decrease in the number of invading HUVECs compared with aspirin or erlotinib alone $(\mathrm{P}<0.01$; ANOVA) (Fig. 5B and C). These observations suggested that aspirin plus erlotinib may inhibit angiogenesis via reducing invasion and migration of endothelial cells. Next, the CAM assay was performed to further investigate the anti-angiogenic effect of aspirin plus erlotinib. As shown in Fig. 5D, aspirin plus erlotinib blocked angiogenesis in the CAM assay. Quantitative analysis revealed that aspirin, erlotinib, and 


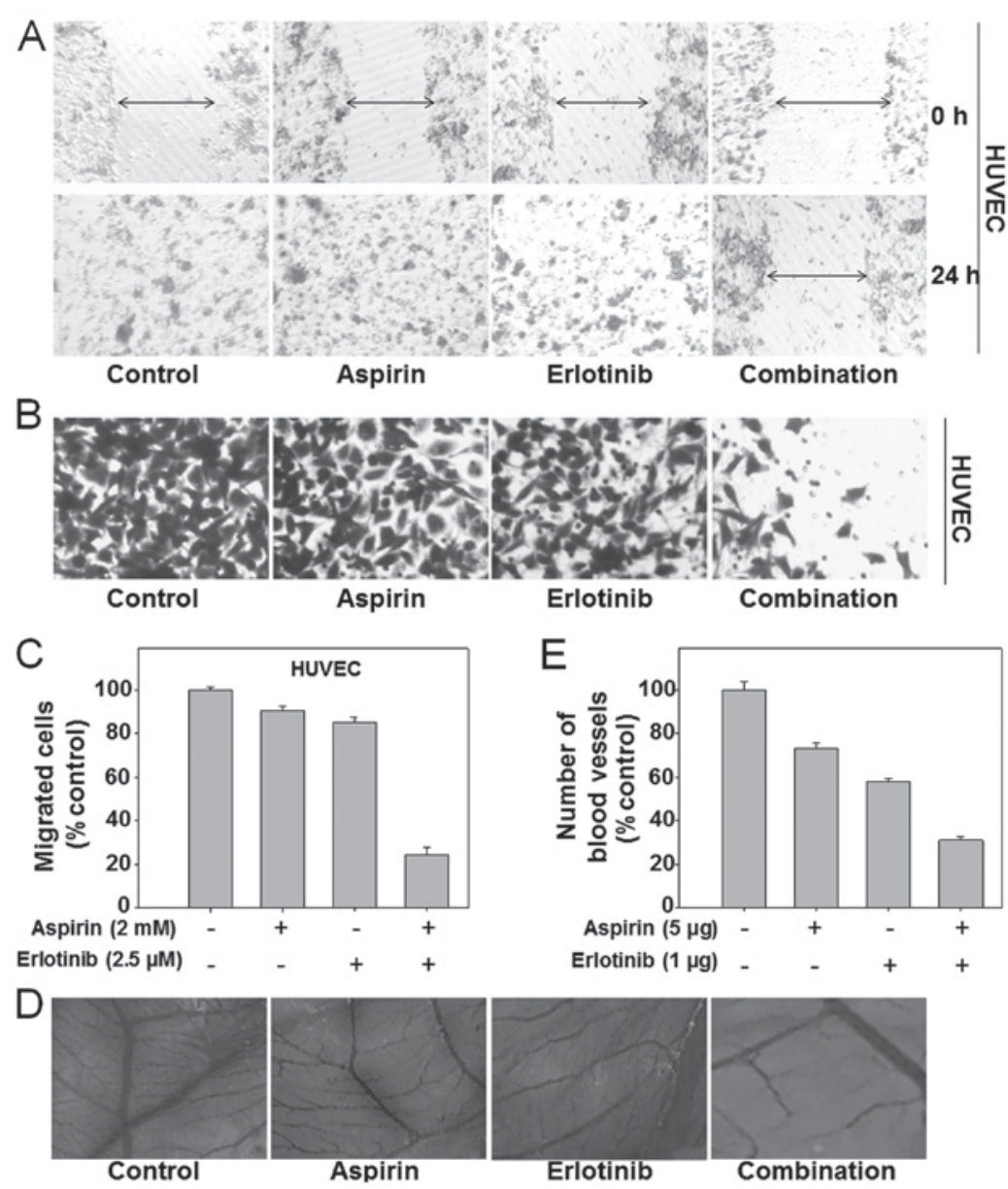

Figure 5. Synergistic anti-angiogenesis by aspirin plus erlotinib. (A) HUVEC cells were gently scratched with a sterile pipette tip and then washed with PBS three times. Next, HUVEC cells were incubated with $2 \mathrm{mM}$ aspirin, $2.5 \mu \mathrm{M}$ erlotinib or the combination for $24 \mathrm{~h}$. Photographs were taken by microscope. (B) HUVEC cells were seeded in the upper chamber and then cells were incubated with $2 \mathrm{mM}$ aspirin, $2.5 \mu \mathrm{M}$ erlotinib or the combination for $24 \mathrm{~h}$. Cell invasion experiments were performed. (C) Quantification of invaded HUVEC cells was counted in Matrigel Transwell invasion assays. (D) CAMs were incubated with aspirin $(5 \mu \mathrm{g})$ and/or erlotinib $(1 \mu \mathrm{g})$ for $48 \mathrm{~h}$. Inhibitory effect of aspirin plus erlotinib on angiogenesis was detected. (E) Blood vessel branches were counted to quantify angiogenesis.

aspirin plus erlotinib caused a $26.7,42.2$ and $68.9 \%$ reduction in the number of blood vessels, respectively $(\mathrm{P}<0.01$; ANOVA) (Fig. 5E). Our data obtained from the three models were sufficient to confirm the synergistic anti-angiogenic activity of aspirin plus erlotinib.

Synergistic antitumor activity of aspirin and erlotinib in vivo. An A549 xenograft model was constructed to verify the anticancer and anti-metastatic efficacy of aspirin plus erlotinib. As demonstrated in Fig. 6A and Table I, aspirin exerted no significant anticancer effect [mean relative tumor volume (RTV) $)_{\text {aspirin }}: 13.3$ vs. mean $\mathrm{RTV}_{\text {control }}: 14.2 ; \mathrm{P}>0.05$, t-test]. Erlotinib exerted a moderate anticancer effect (mean $\mathrm{RTV}_{\text {erlotinib }}: 9.7$ vs. mean $\mathrm{RTV}_{\text {control }}: 14.2, \mathrm{P}<0.05$, t-test). Aspirin plus erlotinib induced significant tumor growth inhibition (mean $\mathrm{RTV}_{\text {combination: }}: 3.1$ vs. mean $\mathrm{RTV}_{\text {control }}: 14.2$, $\mathrm{P}<0.01$, t-test), which was markedly higher compared with that of either aspirin or erlotinib alone (mean $\mathrm{RTV}_{\text {combination }}$ : 3.1 vs. mean $\mathrm{RTV}_{\text {aspirin }}: 13.3, \mathrm{P}<0.05$, t-test and mean $\mathrm{RTV}_{\text {combination }}$ : 3.1 vs. mean $\mathrm{RTV}_{\text {erlotinib }}$ : 9.7, $\mathrm{P}<0.05$, t-test). In addition, the loss of body weight did not differ significantly on day 29 compared with day 0 in the aspirin plus erlotinib group (P>0.05; ANOVA) (Fig.6B). The TUNEL assay was performed to detect the apoptosis induced by aspirin plus erlotinib in the A549 xenograft model. The number of TUNEL-positive cells increased in the tumor tissues of mice receiving the combination treatment (Fig. 6C). Furthermore, caspase activation and XIAP inhibition were observed in the tumor tissues of mice receiving combination treatment, highlighting that apoptosis was involved in the tumor growth inhibitory effects induced by aspirin and erlotinib in vivo (Fig. 6D). In addition, the number of liver metastases was significantly reduced in the combination-treated group compared with the aspirin or erlotinib alone group in the A549 xenograft model $(\mathrm{P}<0.01$; ANOVA) (Fig. 6E). In conclusion, the synergistic antitumor effects of aspirin plus erlotinib were confirmed in vivo.

\section{Discussion}

Cancer cells are sensitive to aspirin in patients with PIK3CA mutation, but not in those with PIK3CA wild-type tumors $(35,36)$. Our data indicated that aspirin plus erlotinib exerted synergistic anti-proliferative effects on PIK3CA wild-type cancer cell lines (A549, NCI-H1299 and HO-8910) and PIK3CA mutant cell lines (HCT-116 and SGC-7901), indicating that PIK3CA mutation was not associated with the 
Table I. In vivo efficacy of aspirin in combination with erlotinib against A549 xenografts.

\begin{tabular}{|c|c|c|c|c|c|c|c|}
\hline \multirow[b]{2}{*}{ Group } & \multicolumn{2}{|c|}{ No. of animals } & \multicolumn{2}{|c|}{ Body weight (g) } & \multirow[b]{2}{*}{ Inhibition rate $(\%)$} & \multirow[b]{2}{*}{ Mean RTV } & \multirow[b]{2}{*}{$\mathrm{T} / \mathrm{C}(100 \%)$} \\
\hline & Start & End & Start & End & & & \\
\hline Control & 6 & 6 & $18.7 \pm 1.5$ & $22.1 \pm 2.7$ & - & 14.2 & - \\
\hline Aspirin (100 mg/kg) & 6 & 6 & $18.6 \pm 1.0$ & $21.5 \pm 1.1$ & 0 & 13.3 & 93.7 \\
\hline Erlotinib (20 mg/kg) & 6 & 6 & $18.7 \pm 1.0$ & $21.1 \pm 0.8$ & 19.2 & $9.7^{\mathrm{a}}$ & 68.3 \\
\hline Combination & 6 & 6 & $19.2 \pm 0.5$ & $22.6 \pm 0.8$ & 55.8 & $3.1^{\mathrm{b}-\mathrm{d}}$ & 21.8 \\
\hline
\end{tabular}

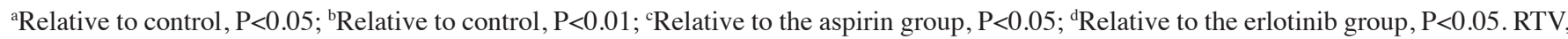
relative tumor volume; T/C, RTV of treated group/mean RTV of control group.

A

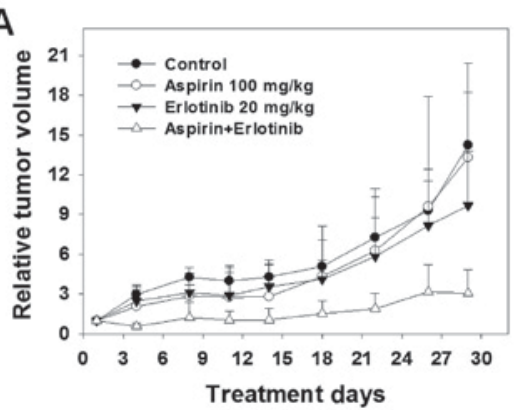

C

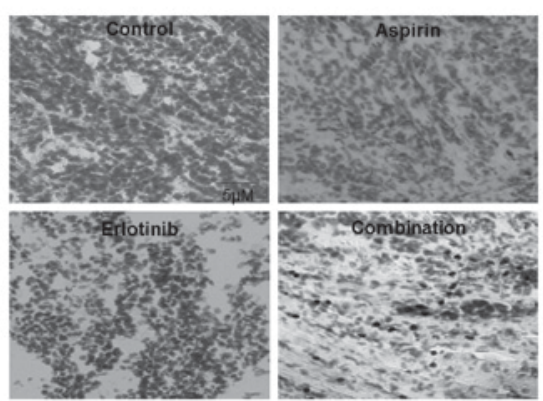

F Aspirin Erlotinib
B

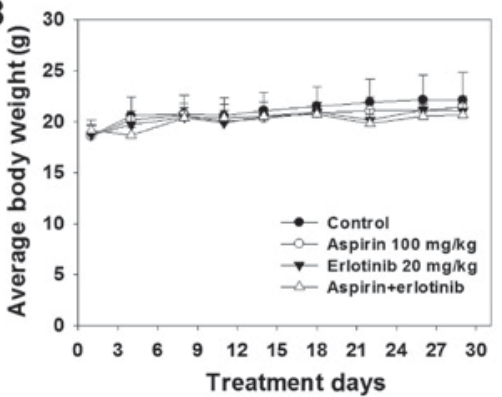

D

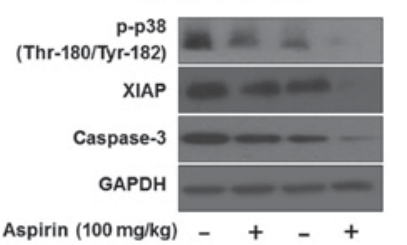

Erlotinib $(20 \mathrm{mg} / \mathrm{kg}) \quad-\quad++$

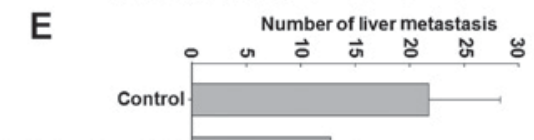

Aspirin $(100 \mathrm{mg} / \mathrm{kg})$

Erlotinib $(20 \mathrm{mg} / \mathrm{kg})$

Aspirinterlotinb

$\longrightarrow$ Apoptosis

E-cadherin

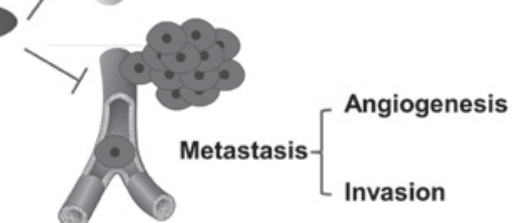

Figure 6. Anticancer efficacy of aspirin and/or erlotinib in vivo. (A) The mice transplanted with A549 human xenografts were randomly divided into 4 groups and given injection of aspirin $(100 \mathrm{mg} / \mathrm{kg})$, erlotinib $(20 \mathrm{mg} / \mathrm{kg})$, combination, or vehicle for a period of 29 days. RTVs were expressed as mean \pm SD ( $\mathrm{n}=6)$. (B) The average body weight was expressed as mean $\pm \mathrm{SD}(\mathrm{n}=6)$. (C) Apoptosis was detected by TUNEL staining in A549 xenografts experiment. (D) The tumors ( $\mathrm{n}=6$ per group) were weighed and mixed, the levels of Protein extracted from tumor tissues in A549 xenografts experiment were evaluated by western blotting. (E) Metastases to the liver were detected in a histologic slide. Each bar in the graph represents the average number of liver metastasis in each experimental group, ten histologically similar fields were randomly selected from each slide for analysis. (F) Conclusion for synergistic anticancer activity by combining aspirin with erlotinib. RTV, mean relative tumor volume.

synergistic anti-proliferative effect exerted by aspirin plus erlotinib on human cancer cells. Thus, aspirin plus erlotinib may improve the antitumor efficacy both in aspirin-sensitive and aspirin-resistant cancer cells. The co-administration of aspirin and erlotinib markedly improved the antitumor efficacy without increasing the toxicity in a A549 xenograft model in vivo.

EGFR-TKIs, such as afatinib, gefitinib and erlotinib, have been proven to be clinically effective for patients with 
metastatic or locally advanced non-small cell lung cancer (NSCLC) (37). However, treatment with EGFR-TKIs eventually fails due to the development of acquired drug resistance within 9-14 months of treatment (38). EGFR-TKI-resistant NSCLC cells undergo EMT by repressing E-cadherin and upregulating p38 expression (39). The loss of E-cadherin expression and upregulation of $\mathrm{p} 38$ activate multiple pathways that inhibit apoptosis, induce tumor metastasis and erlotinib resistance, and eventually lead to failure of erlotinib treatment $(30,40)$. Thus, overexpression of E-cadherin and inhibition of p38 may be effective in overcoming EMT induction and apoptosis evasion-mediated erlotinib resistance. Our data indicated that aspirin plus erlotinib significantly induced mitochondrial-mediated apoptosis, and significantly induced the activation of E-cadherin and suppression of p38 both in A549 and SGC-7901 cells. Moreover, elevated expression of E-cadherin increased apoptosis induced by aspirin plus erlotinib; in addition, the inhibition of p38 by SB-203580 or siRNA also enhanced the apoptosis induced by aspirin plus erlotinib. These data indicated that the $\mathrm{p} 38 / \mathrm{E}$-cadherin signaling pathway was implicated in the enhanced apoptosis induced by aspirin plus erlotinib, and aspirin may reverse erlotinib resistance via the $\mathrm{p} 38 / \mathrm{E}$-cadherin pathway.

As p38 and E-cadherin also play a key role in EMT and tumor metastasis, we hypothesized that aspirin plus erlotinib may significantly inhibit EMT and the metastatic process. EMT has been shown to play a crucial role in the invasion and metastasis of epithelial tumors, as it enables cancer cell migration and invasion (41). Our data indicated that combined treatment with aspirin and erlotinib enhanced the inhibition on cancer cell migration and invasion. Metastasis and tumor growth depend on the development of a neovasculature around and in the tumor (42). Angiogenesis is a neovascularization process that involves critical steps, including endothelial cell invasion, migration and proliferation (43). Our results determined that aspirin plus erlotinib inhibited angiogenesis by suppressing migration and invasion of endothelial cells. Furthermore, the synergistic anti-angiogenic effects of aspirin plus erlotinib were confirmed by the CAM assay. Most importantly, aspirin plus erlotinib significantly decreased the number of liver metastases in vivo. Overall, these findings demonstrated that aspirin plus erlotinib significantly inhibited tumor metastasis via inhibiting EMT and angiogenesis; thus, aspirin plus erlotinib may be an efficient combination regimen for patients with metastatic cancer.

In summary, to the best of our knowledge, our data are the first to demonstrate that combining aspirin with erlotinib could significantly inhibit the proliferation and induce apoptosis of human cancer cells. In addition, our data indicated that aspirin and erlotinib inhibited EMT and angiogenesis, consequently suppressing tumor metastasis. Furthermore, the p38/E-cadherin signaling pathway was involved in the synergistic anticancer activity of aspirin plus erlotinib (Fig. 6F). Therefore, aspirin appears to be a pertinent sensitizer to erlotinib for treating patients with metastatic cancer.

\section{Acknowledgements}

Not applicable.

\section{Funding}

The present study was funded by National Natural Science Foundation of China (grant no. 1702887), Hangzhou Major Science and Technology Project (grant no. 20172016A01), Fund of Hangzhou Medical Key Discipline Construction (grant no. 2017-51-07), Zhejiang Provincial Foundation of Natural Science (grant no. LQ16H310004), Public-service Technology Research Plan of Zhejiang Province (grant nos. 2015C33269 and 2016C33210), High-level Talents Coming Back from Abroad Innovation and Entrepreneurship Program in Hangzhou (grant no. 2051), Zhejiang Provincial Program for the cultivation of high-level innovative health talents (grant no. 2010-190-4), Scientific and Technological Developing Scheme of Hangzhou City (grant nos. 20150733Q14 and 20140633B03).

\section{Availability of data and materials}

The datasets used and/or analyzed during the present study are available from the corresponding author on reasonable request.

\section{Authors' contributions}

$\mathrm{CZ}$ and NML were responsible for the conception and design of the study. XH and XW were responsible for collecting the data. LWW analyzed and interpreted the data. CZ drafted this manuscript. NML revised it critically for important intellectual content. All authors read and approved the final manuscript.

\section{Ethics approval and consent to participate}

All animal handling was performed in accordance with the National Institutes of Health Guide for the Care and Use of Laboratory Animals, and approved by the Zhejiang University City College Animal Care and Use Committee.

\section{Consent for publication}

Not applicable.

\section{Competing interests}

The authors declare no competing interests.

\section{References}

1. Khan P, Manna A, Saha S, Mohanty S, Mukherjee S, Mazumdar M, Guha D and Das T: Aspirin inhibits epithelial-to-mesenchymal transition and migration of oncogenic K-ras-expressing non-small cell lung carcinoma cells by down-regulating E-cadherin repressor Slug. BMC Cancer 16: 39, 2016.

2. Kurimoto R, Iwasawa S, Ebata T, Ishiwata T, Sekine I, Tada Y, Tatsumi K, Koide S, Iwama A and Takiguchi Y: Drug resistance originating from a TGF- $\beta /$ FGF-2-driven epithelial-to-mesenchymal transition and its reversion in human lung adenocarcinoma cell lines harboring an EGFR mutation. Int J Oncol 48: 1825-1836, 2016.

3. Matsuda Y, Miura K, Yamane J, Shima H, Fujibuchi W, Ishida K, Fujishima F, Ohnuma S, Sasaki H, Nagao M, et al: SERPINI1 regulates epithelial-mesenchymal transition in an orthotopic implantation model of colorectal cancer. Cancer Sci 107: 619-628, 2016. 
4. Onder TT, Gupta PB, Mani SA, Yang J, Lander ES and Weinberg RA: Loss of E-cadherin promotes metastasis via multiple downstream transcriptional pathways. Cancer Res 68: 3645-3654, 2008.

5. Singh A and Settleman J: EMT, cancer stem cells and drug resistance: An emerging axis of evil in the war on cancer. Oncogene 29: 4741-4751, 2010.

6. Lu M, Marsters S, Ye X, Luis E, Gonzalez L and Ashkenazi A E-cadherin couples death receptors to the cytoskeleton to regulate apoptosis. Mol Cell 54: 987-998, 2014.

7. McConkey DJ, Choi W, Marquis L, Martin F, Williams MB, Shah J, Svatek R, Das A, Adam L, Kamat A, et al: Role of epithelial-to-mesenchymal transition (EMT) in drug sensitivity and metastasis in bladder cancer. Cancer Metastasis Rev 28: 335-344, 2009.

8. Bolos V, Peinado H, Perez-Moreno MA, Fraga MF, Esteller M and Cano A: The transcription factor Slug represses E-cadherin expression and induces epithelial to mesenchymal transitions: A comparison with Snail and E47 repressors. J Cell Sci 116: 499-511, 2003

9. Peinado H, Olmeda D and Cano A: Snail, Zeb and bHLH factors in tumour progression: An alliance against the epithelial phenotype? Nat rev Cancer 7: 415-428, 2007.

10. Wang H, Fang R, Wang XF, Zhang F, Chen DY, Zhou B Wang HS, Cai SH and Du J: Stabilization of Snail through AKT/GSK-3 $\beta$ signaling pathway is required for TNF- $\alpha$-induced epithelial-mesenchymal transition in prostate cancer PC3 cells. Eur J Pharmacol 714: 48-55, 2013.

11. Zohn IE, Li Y, Skolnik EY, Anderson KV, Han J and Niswander L: p38 and a p38-interacting protein are critical for downregulation of E-cadherin during mouse gastrulation. Cell 125: 957-969, 2006.

12. Chen R, Yang Q and Lee JD: BMK1 kinase suppresses epithelial-mesenchymal transition through the Akt/GSK3 $\beta$ signaling pathway. Cancer Res 72: 1579-1587, 2012

13. Cheng JC, Klausen C and Leung PC: Hydrogen peroxide mediates EGF-induced down-regulation of E-cadherin expression via p38 MAPK and snail in human ovarian cancer cells. Mol Endocrinol 24: 1569-1580, 2010

14. Sui X, Kong N, Ye L, Han W, Zhou J, Zhang Q, He C and Pan H: p38 and JNK MAPK pathways control the balance of apoptosis and autophagy in response to chemotherapeutic agents. Cancer Lett 344: 174-179, 2014

15. Olson JM and Hallahan AR: p38 MAP kinase: A convergence point in cancer therapy. Trends Mol Med 10: 125-129, 2004.

16. Limami Y, Pinon A, Leger DY, Pinault E, Delage C, Beneytout JL, Simon A and Liagre B: The P2Y2/Src/p38/COX-2 pathway is involved in the resistance to ursolic acid-induced apoptosis in colorectal and prostate cancer cells. Biochimie 94: 1754-1763, 2012.

17. Salim H, Akbar NS, Zong D, Vaculova AH, Lewensohn R, Moshfegh A, Viktorsson K and Zhivotovsky B: miRNA-214 modulates radiotherapy response of non-small cell lung cancer cells through regulation of p38MAPK, apoptosis and senescence. Br J Cancer 107: 1361-1373, 2012.

18. Yan KH, Yao CJ, Chang HY, Lai GM, Cheng AL and Chuang SE: The synergistic anticancer effect of troglitazone combined with aspirin causes cell cycle arrest and apoptosis in human lung cancer cells. Mol Carcinog 49: 235-246, 2010.

19. Chan AT, Ogino S and Fuchs CS: Aspirin and the risk of colorectal cancer in relation to the expression of COX-2. N Engl J Med 356: 2131-2142, 2007.

20. Bardia A, Keenan TE, Ebbert JO, Lazovich D, Wang AH, Vierkant RA, Olson JE, Vachon CM, Limburg PJ, Anderson KE and Cerhan JR: Personalizing aspirin use for targeted breast cancer chemoprevention in postmenopausal women. Mayo Clin Proc 91: 71-80, 2016.

21. Petrick JL, Sahasrabuddhe VV, Chan AT, Alavanja MC, Beane-Freeman LE, Buring JE, Chen J, Chong DQ, Freedman ND, Fuchs CS, et al: NSAID Use and risk of hepatocellular carcinoma and intrahepatic cholangiocarcinoma: The liver cancer pooling project. Cancer Prev Res 8: 1156-1162, 2015

22. Brasky TM, Baik CS, Slatore CG, Potter JD and White E: Non-steroidal anti-inflammatory drugs and small cell lung cancer risk in the VITAL study. Lung Cancer 77: 260-264, 2012

23. Rothwell PM, Wilson M, Price JF, Belch JF, Meade TW and Mehta Z: Effect of daily aspirin on risk of cancer metastasis: A study of incident cancers during randomised controlled trials. Lancet 379: 1591-1601, 2012.
24. Guillem-Llobat P, Dovizio M, Bruno A, Ricciotti E, Cufino V, Sacco A, Grande R, Alberti S, Arena V, Cirillo M, et al: Aspirin prevents colorectal cancer metastasis in mice by splitting the crosstalk between platelets and tumor cells. Oncotarget 7: 32462-32477, 2016

25. Ito K, Semba T, Uenaka T, Wakabayashi T, Asada $M$ and Funahashi Y: Enhanced anti-angiogenic effect of E7820 in combination with erlotinib in epidermal growth factor receptor-tyrosine kinase inhibitor-resistant non-small-cell lung cancer xenograft models. Cancer Sci 105: 1023-1031, 2014.

26. Hirai F, Edagawa M, Shimamatsu S, Toyozawa R, Toyokawa G, Nosaki K, Yamaguchi M, Seto T, Takenoyama M and Ichinose Y: Evaluation of erlotinib for the treatment of patients with non-small cell lung cancer with epidermal growth factor receptor wild type. Oncol Lett 14: 306-312, 2017.

27. Li YL, Sun J, Hu X, Pan YN, Yan W, Li QY, Wang F, Lin NM and Zhang C: Epothilone B induces apoptosis and enhances apoptotic effects of ABT-737 on human cancer cells via PI3K/AKT/mTOR pathway. J Cancer Res Clin Oncol 142: 2281-2289, 2016

28. Zhang C, Shi J, Mao SY, Xu YS, Zhang D, Feng LY, Zhang B, Yan YY, Wang SC, Pan JP, et al: Role of p38 MAPK in enhanced human cancer cells killing by the combination of aspirin and ABT-737. J Cell Mol Med 19: 408-417, 2015.

29. Zhang C, Cai TY, Zhu H, Yang LQ, Jiang H, Dong XW, Hu YZ, Lin NM, He QJ and Yang B: Synergistic antitumor activity of gemcitabine and ABT-737 in vitro and in vivo through disrupting the interaction of USP9X and Mcl-1. Mol Cancer Ther 10: 1264-1275, 2011

30. Fernando RI, Hamilton DH, Dominguez C, David JM, McCampbell KK and Palena C: IL-8 signaling is involved in resistance of lung carcinoma cells to erlotinib. Oncotarget 7: 42031-42044, 2016.

31. Witta SE, Gemmill RM, Hirsch FR, Coldren CD, Hedman K, Ravdel L, Helfrich B, Dziadziuszko R, Chan DC, Sugita M, et al: Restoring E-cadherin expression increases sensitivity to epidermal growth factor receptor inhibitors in lung cancer cell lines. Cancer Res 66: 944-950, 2006.

32. Yauch RL, Januario T, Eberhard DA, Cavet G, Zhu W, Fu L, Pham TQ, Soriano R, Stinson J, Seshagiri S, et al: Epithelial versus mesenchymal phenotype determines in vitro sensitivity and predicts clinical activity of erlotinib in lung cancer patients. Clin Cancer Res 11: 8686-8698, 2005.

33. Heerboth S, Housman G, Leary M, Longacre M, Byler S, Lapinska K, Willbanks A and Sarkar S: EMT and tumor metastasis. Clin Transl Med 4: 6, 2015.

34. Bhat TA, Nambiar D, Tailor D, Pal A, Agarwal R and Singh RP: Acacetin inhibits in vitro and in vivo angiogenesis and downregulates Stat signaling and VEGF expression. Cancer Prev Res 6: 1128-1139, 2013.

35. Liao X, Lochhead P, Nishihara R, Morikawa T, Kuchiba A, Yamauchi M, Imamura Y, Qian ZR, Baba Y, Shima K, et al: Aspirin use, tumor PIK3CA mutation and colorectal-cancer survival. N Engl J Med 367: 1596-1606, 2012.

36. Turturro SB, NajorMS, Ruby CE, Cobleigh MA and Abukhdeir AM: Mutations in PIK3CA sensitize breast cancer cells to physiologic levels of aspirin. Breast Cancer Res Treat 156: 33-43, 2016.

37. Burotto M, Manasanch EE, Wilkerson J and Fojo T: Gefitinib and erlotinib in metastatic non-small cell lung cancer: A meta-analysis of toxicity and efficacy of randomized clinical trials. Oncologist 20: 400-410, 2015

38. Zhang B, Jiao J, Liu Y, Guo LX, Zhou B, Li GQ, Yao ZJ and Zhou GB: Gefitinib analogue induces apoptosis of T790M EGFR-harboring lung cancer cells by up-regulation of the BH-3 only protein Noxa. PLoS One 7: e48748, 2012.

39. Rastogi I, Rajanna S, Webb A, Chhabra G, Foster B, Webb B and Puri N: Mechanism of c-Met and EGFR tyrosine kinase inhibitor resistance through epithelial mesenchymal transition in non-small cell lung cancer. Biochem Biophys Res Commun 477: 937-944, 2016.

40. Wang Y, Sheng Q, Spillman MA, Behbakht K and Gu H: Gab2 regulates the migratory behaviors and E-cadherin expression via activation of the PI3K pathway in ovarian cancer cells. Oncogene 31: 2512-2520, 2012.

41. Yang $\mathbf{J}$ and Weinberg RA: Epithelial-mesenchymal transition: At the crossroads of development and tumor metastasis. Dev Cell 14: 818-829, 2008.

42. Jain RK: Normalization of tumor vasculature: An emerging concept in antiangiogenic therapy. Science 307: 58-62, 2005.

43. Lamalice L, Le Boeuf F and Huot J: Endothelial cell migration during angiogenesis. Circ Res 100: 782-794, 2007. 